\title{
CALIDAD DEL SUEÑO Y SU ASOCIACIÓN CON EL RENDIMIENTO ACADÉMICO DE LOS PROGRAMAS DE PREGRADO DE LA INSTITUCIÓN UNIVERSITARIA COLEGIOS DE COLOMBIA - UNICOC, SEDE BOGOTÁ.
}

\author{
BEHAVIORS OF DREAM AND ITS ASSOCIATION WITH THE ACADEMIC PERFORMANCE OF THE \\ UNDERGRADUATE PROGRAMS OF THE UNIVERSITY INSTITUTION COLLEGES OF COLOMBIA - UNICOC, \\ BOGOTA HEADQUARTERS.
}

Aguilera Rojas Sandra Elizabeth*, Bicenty Mendoza Ángela*, Ibáñez Pinilla Édgar Antonio*, Marentes

Delgado Adriana*, Cruz Jiménez Valentina*, Ruiz García Luz Amparo*.

* Institución Universitaria Colegios de Colombia UNICOC Sede Bogotá. Colombia.

Citation: Aguilera Rojas SE., Bicenty Mendoza A., Ibáñez Pinilla EA., Marentes Delgado A., Cruz Jiménez V., Ruiz García LA. (2020) Calidad del sueño y su Asociación con el Rendimiento Académico de los Programas de Pregrado de la Institución Universitaria Colegios de Colombia - UNICOC, Sede Bogotá. Revista Salud Pública y Nutrición, 19 (2), 26-35.

Editor: Esteban G. Ramos Peña, Dr. CS., Universidad Autónoma de Nuevo León, Facultad de Salud Pública y Nutrición, Monterrey Nuevo León, México. Copyright: (C2020 Aguilera Rojas SE. et al. This is an open-access article distributed under the terms of Creative Commons Attribution License [CC BY 4.0], which permits unrestricted use, distribution, and reproduction in any medium, provided the original author and source are credited.

Competing interests: The authors have declared that no competing interests exist.

DOI: https://doi.org/10.29105/respyn19.2-4

Recibido: 31 de diciembre 2019; Aceptado: 01 de junio 2020

Email: lagarcia@unicoc.edu.co 


\title{
CALIDAD DEL SUEÑO Y SU ASOCIACIÓN CON EL RENDIMIENTO ACADÉMICO DE LOS PROGRAMAS DE PREGRADO DE LA INSTITUCIÓN UNIVERSITARIA COLEGIOS DE COLOMBIA - UNICOC, SEDE BOGOTÁ.
}

\author{
Aguilera Rojas Sandra Elizabeth*, Bicenty Mendoza Ángela*, Ibáñez Pinilla Édgar Antonio*, Marentes Delgado \\ Adriana*, Cruz Jiménez Valentina*, Ruiz García Luz Amparo*. \\ * Institución Universitaria Colegios De Colombia UNICOC Sede Bogotá. Colombia.
}

\begin{abstract}
RESUMEN
Introducción. El sueño es un estado fisiológico que es transitorio y periódico. Y se define como una necesidad básica del ser humano. Objetivo: Establecer la relación entre la calidad del sueño y el rendimiento académico de los alumnos de los programas de pregrado de la institución universitaria Colegios de Colombia UNICOC. Material y Método: Estudio descriptivo de corte transversal. La muestra estuvo compuesta por 266 estudiantes universitarios. Se incluyeron variables sociodemográficas, clínicas y académicas. Se utilizó la prueba chi cuadrado de Pearson y modelo de regresión logística con el método Enter. (SPSS v22). Para la obtención de datos se aplicó el índice de calidad del Sueño de Pittsburg (PSG) y la escala de somnolencia Epworth (ESE). Resultados: El promedio del índice de PSG fue de $8,7 \pm 3$ y el de ESE fue 9,09 \pm 4.07 Entre el PSG y el ESE hubo asociación estadísticamente significativa $(p=0,004)$. Conclusiones: Se concluye que el rendimiento académico está asociado a la mala calidad del sueño y la somnolencia las cuales fueron prevalentes, especialmente en los estudiantes del Colegio Odontológico.

Palabras Clave: Disomnias, desórdenes de excesiva somnolencia, desarrollo de desórdenes académicos, aprendizaje.

\section{ABSTRACT}

Introduction: Sleep is a physiological state that is transient and periodic. And it is defined as a basic need of the human being. Objective: To establish the relationship between the quality of sleep and the academic performance for the students of the undergraduate programs at the university institution Colleges of Colombia - UNICOC. Material and method: A cross-sectional study that includes 266 students. Type of sampling was stratified by proportional allocation the applied instruments were Pittsburgh Scale (ICSP) and Somnolence Epworth, in addition to sociodemographic, clinical and academic variables included. Pearson's chi-square test and logistic regression model with the Enter method were used. SPSS v22. Results: The mean sleep quality index (PSG) was $8.7 \pm 3$ and ESE was $9,09 \pm 4.07$. Between PSG and ESE, there was statistically significant association ( $p$ $=0.004)$. It is concluded that the academic performance is associated to the poor quality of sleep and drowsiness which were prevalent, especially in the students of the Odonatological College.
\end{abstract}

Key words: Dyssomnias, Disorders of Excessive Somnolence, Developmental Academic Disorder, learning. 


\section{Introducción}

El sueño es un estado fisiológico fundamental que se caracteriza por ser transitorio, periódico, reversible e ineludible en la vida del ser humano. Se destaca por su función importante en el Sistema Nervioso, al estar intrínsecamente relacionado con las vías de secreción hormonal y las del dolor. Por consiguiente, Maslow lo define como una "necesidad básica del ser humano" (Lezcano H, 2014). El sueño, así como el descanso son fundamentales para tener una buena salud y así lograr mejorar la calidad de vida. Los efectos del sueño no se limitan al propio organismo, con la necesidad de restauración neurológica, sino que, afectan al desarrollo y funcionamiento normal de las capacidades cognoscitivas e intelectuales de las personas (Bohorquez, 2011). Por tal motivo se ha estandarizado que: el número de horas adecuadas para que una persona consiga descansar y reponerse de las actividades cotidianas oscila entre 7 y 9 horas. En estados donde hay un menor número de 6 horas de sueño se genera una acumulación progresiva de cansancio, que produce estados de fatiga, por consiguiente, disminución de la calidad de vida. Además, en casos más extremos se registran alteraciones del comportamiento, atención y falta de memoria (Cañones P.J, 2003), debido a que el sueño actúa en el cerebro como el disco duro de un computador (Lezcano H, 2014).

Estudios han reportado que individuos con trastornos y mala calidad del sueño presentan un riesgo elevado de desarrollar patologías como: hipertensión arterial, obesidad, síndrome metabólico y diabetes (Grander M.A, 2012). Por otro lado, en el caso de la población joven universitaria, autores como Lund Reider en el 2010, encontraron cambios en los patrones de sueño cuando los adolescentes ingresan a la universidad. Estas alteraciones son capaces de producir dificultad para la concentración dentro del aula de clases que genera un aprendizaje no significativo. En estudios realizados con estudiantes universitarios se ha encontrado que estos presentan mala calidad del sueño, como consecuencia de factores académicos, socio económicos, familiares o de tipo personal. En el estudio realizado por Lezcano, Vieto, Morán, Donadío \& Carbonó (2014), con estudiantes de medicina de la universidad de Panamá cuyo objetivo era determinar las características y la calidad de sueño se concluye que, de una muestra de 290 estudiantes, según la escala de somnolencia y el índice de calidad del sueño el $67.6 \%$ son malos dormidores lo que influye directamente con la calidad del sueño. La universidad de Quintana Roo en la ciudad de México buscó determinar los factores que inciden en la calidad del sueño, encontrando que la falta de sueño se relaciona con factores como el consumo de alcohol, café y tabaco, a su vez se buscó la relación entre la calidad del sueño y el rendimiento académico indicando que existe la posibilidad de que la mala calidad del sueño pueda afectar el rendimiento académico de los estudiantes. (Aguirre., 2014).

De la misma forma, en Colombia se han realizado estudios buscando evaluar la mala calidad del sueño y conductas de sueño. Un ejemplo, es el de Machado realizado en 2015 con estudiantes de Medicina, quien reportó que existe una alta frecuencia de somnolencia diurna cercana al $60 \%$ además, de una mala calidad de sueño siendo con un predominante del $80 \%$, lo anterior, debido a los horarios de los estudiantes en los que se presenta una disrupción del ritmo día/noche. En adición, otro estudio realizado en la Universidad Nacional reporta que, más de la mitad de los estudiantes (66\%) tienen mala higiene del sueño, lo cual provoca afectaciones a la hora dormir como la interrupción continua del patrón del sueño, convirtiéndose en un mal hábito. A demás, en este estudio también se buscó establecer la relación de los hábitos de sueño con el rendimiento académico, encontrando que; no fue tan fuerte dicha asociación a pesar de la alta prevalencia de falta de sueño, pero si se pudo asociar que los estudiantes con mejor rendimiento académico son en mayoría malos dormilones (Escobar - Cordoba, 2011). En estudio realizado en la Universidad de Cartagena con 210 estudiantes de la facultad de medicina se determina que 185 son caracterizados como malos dormidores y 25 como buenos dormidores, situación que está relacionada con la calidad del sueño y el estado de somnolencia, se observaron diferencias significativas en cuanto al rendimiento académico de los dos grupos. (Monterrosa Castro, 2014). Caicedo Ochoa, en su estudio de prevalencia del grado de somnolencia y su relación con el rendimiento académico en estudiantes de medicina de la Ciudad de Tunja, concluyendo que La prevalencia de somnolencia es alta en estudiantes de Medicina y su relación con el rendimiento académico no se evidencia en la población estudiada (Caicedo Ochoa Y., 2016). En estudio realizado en una Universidad Pública de la ciudad de Manizales, cuyo objetivo fue 
determinar la calidad del sueño y la somnolencia diurna excesiva en universitarios de diferentes carreras de pregrado, se concluyó que la mala calidad del sueño y la somnolencia se presentan en todas las áreas del conocimiento y que no son de exclusividad de disciplinas del sector salud. (de la Portilla Maya S, 2019)

La mala calidad del sueño trae consigo trastornos de sueño (TS) siendo estos una de las principales causas de consulta médica, además de, morbilidad física y mental, que pueden afectar a individuos de cualquier edad o condición socioeconómica. Sin embargo, existe poca información sobre su prevalencia y repercusión en diferentes aspectos de la vida de las personas. En Colombia no se han realizado suficientes investigaciones en este campo y, por tanto, se carece de información propia para implementar políticas de prevención y manejo (Amador J, 2015). El sueño tiene una importancia relevante en los procesos de consolidación de la memoria, aprendizaje, procesos restaurativos y codificación (Veldi M, 2005). Por ello, es importante la buena calidad del sueño en estudiantes universitarios. Los disturbios del sueño o la mala calidad del sueño se asocian a reducción en la vitalidad, mal funcionamiento social y deterioro en la actividad física, salud mental, procesos cognitivos, rendimiento sicomotor y calidad de vida (Ahrberg K, 2012) (Curcio G, 2006). El insomnio tiene tasas de prevalencia en estudiantes universitarios del 69,0\% (Lund HG, 2010, Preisegolaviciute E, 2010). y se ha asociado con estados de mayor fatiga, irritabilidad, ansiedad, depresión, dificultad para terminar las tareas, déficit cognitivo, accidentes, ausentismo, o dependencia de sustancias, suicidio, compromiso inmunológico, enfermedades cardiovasculares y gastrointestinales (Cheng SH, 2012).

Por otro lado, es bien conocida la importancia de una correcta interacción entre memoria, aprendizaje y codificación, pero estos deben estar acompañados de una buena calidad del sueño, lo cual permitirá el logro y materialización de los proyectos de vida (Escobar - Cordoba, 2011, Amador J, 2015, Veldi M, 2005). Cuando hay deficientes resultados académicos, estos repercuten en otros ámbitos como el social que conduce a exclusión familiar, económicos por la carga que este genera, pero es importante señalar que no sólo se afecta el individuo como persona, sino que esto también incide en la Institución formadora ya que los tiempos y momentos programados se retrasan y generan costos adicionales. (Veldi M, 2005)

Malos Hábitos del Sueño en estudiantes adolescentes, así como las largas jornadas de estudio, las prácticas asistenciales en las clínicas, y los trabajos de laboratorio de preclínica, en el caso específico de estudiantes de Odontología, podrían afectar de forma negativa el sueño, debido a la privación de este, lo cual conllevaría a situaciones de somnolencia diurna y diminución de la capacidad de respuesta frente a las situaciones cotidianas. Esto sin contar que existen factores externos asociados a un mal dormir tales como el consumo de cigarrillo, alcohol, el sedentarismo, uso excesivo de internet, por citar algunos. Es importante que las instituciones de educación superior (IES), sean capaces de reconocer señales tales como cambios en la conducta, adecuada actitud resolutiva frente a los problemas por parte de sus estudiantes, ya que esto indiscutiblemente conlleva a un deterioro en la actividad física, los procesos cognitivos, el rendimiento psicomotor y la calidad de vida (Ahrberg K, 2012, Curcio G, 2006).

El rendimiento académico de los estudiantes universitarios constituye un factor imprescindible y fundamental para la valoración de la calidad educativa en la enseñanza superior. (Enríquez Guerrero, Segura Cardona, \& Cuevas, 2013) Su carácter complejo y multidimensional evoluciona a partir de concepciones centradas en el alumno (basadas en la voluntad o en la capacidad de este), en los resultados de su trabajo escolar, hasta concepciones holísticas que atribuyen el rendimiento a un conjunto de factores derivados del sistema educativo, de la familia y del propio alumno. El bajo rendimiento académico, es el resultado de múltiples factores y causas, entre los que se encuentran: los de carácter individual con relación a su estado de salud, el contexto familiar, la situación socioeconómica y otros asociados al propio sistema educativo.

En una investigación realizada por Pérez, Ramón, Sánchez (2000) con estudiantes universitarios demuestra, que la motivación está asociada a la asistencia a clases, y que la ausencia a las lecciones se relaciona con problemas de repetición y abandono a los estudios. Cuanta mayor asistencia, mejor 
calificación; la asistencia es una de las variables más significativas que influye en el rendimiento académico del alumno. Conclusiones similares las encontró Montero y Villalobos (2004) en un estudio realizado con universitarios de la universidad de Costa Rica, en la que se evidenció que los estudiantes y las estudiantes que asisten a clases regularmente siempre o casi siempre tienen en promedio 0,26 puntos más que aquello estudiantes que no lo hacen en forma regular. (Enríquez Guerrero, Segura Cardona, \& Cuevas, 2013).

El solo hecho de que una institución pueda llegar a tener control en las faltas de sus estudiantes a clase, y determinar que no fueron por razones como, qué necesitaban dormir más o por múltiples causas, podrían ayudar un estudiante a tener mejor rendimiento académico, mejora en la planificación de su tiempo, y tan solo con crear políticas preventivas institucionales, se generaría un impacto en la calidad de vida de un futuro profesional y a su vez se generan herramientas para responder a las exigencias y demandas educativas en un contexto marcado por desafíos propios del proceso de la globalización hacia la búsqueda de la alta calidad institucional.

Por las anteriores razones se cree que la calidad del sueño y los malos hábitos del sueño, en estudiantes adolescentes, las largas jornadas de estudio de los estudiantes de los colegios Administrativo, Jurídico y Odontológico, así como las prácticas asistenciales en las clínicas, o trabajos de laboratorio de preclínica, en el caso específico de estudiantes de Odontología, podrían afectar de forma negativa el sueño, lo cual conllevaría a situaciones de somnolencia diurna lo que afectaría su rendimiento académico. De tal forma que el presente trabajo se planteó como objetivo determinar la calidad del sueño y su asociación con el rendimiento académico de los estudiantes de pregrado de la Institución Universitaria Colegios de Colombia - UNICOC, sede Bogotá.

\section{Material y Método}

El tipo de estudio fue observacional, descriptivo, de corte transversal en una población de estudiantes de pregrado matriculados en el primer periodo de 2016 de la Institución Universitaria Colegios de Colombia - UNICOC, sede Bogotá, conformada por tres colegios: Administrativo, Jurídico y Odontológico.
La muestra estuvo conformada por 266 estudiantes: 25 estudiantes del colegio Jurídico, 32 estudiantes del colegio Administrativo y 209 estudiantes del colegio Odontológico. A los que se les aplicaron los instrumentos índices de Pittsburg (PSG) y la escala de somnolencia de Epworth (ESE), previa firma del consentimiento informado. Como criterios de inclusión se tomaron estudiantes que aceptaron participar voluntariamente en el estudio y como exclusión estudiante que no se encontraban el día de la aplicación del instrumento. El tipo de muestreo fue estratificado por afijación proporcional para los colegios administrativo y odontológico no probabilístico y censal para la población del colegio jurídico; el tamaño de la muestra se calculó con una prevalencia del $50 \%$, una precisión del $6 \%$ y una confiabilidad del $95 \%$ para una total de 215 estudiantes, estimando una pérdida del $10 \%$ y por aproximaciones el tamaño fue de 241 estudiantes de los colegio administrativo y odontológico, y 25 estudiantes del colegio jurídico para una total de 266 estudiantes.

Se tomaron variables como edad en años, sexo, condición socioeconómica, estado civil, lugar de procedencia, peso, talla, IMC. La variable dependiente objeto de estudio fue el rendimiento académico, definida como número de asignaturas reprobadas en el semestre. Para determinar índice de calidad del sueño se utilizó la escala Pittsburgh (ICSP)instrumento que consta de diecinueve preguntas de autoevaluación y cinco preguntas dirigidas a los compañeros de prueba, el instrumento se distribuye en siete categorías a saber: calidad subjetiva de sueño, latencia, duración, eficiencia, perturbaciones del sueño, uso de medicación para dormir, disfunción diurna (Honorio \& Hideyo, 2013), la finalidad evaluar la calidad global y sus perturbaciones en el último mes; la puntuación de las todas las áreas se suman para dar una puntuación global que oscilará entre 0 y 21 puntos, donde el " 0 " indica facilidad para dormir y " 21 " dificultad severa en todas las áreas, por lo que se deriva la siguiente escala de puntuación: de 0 a 5 se considera como "buenos dormidores", mayor de 5 una mala calidad del sueño (Royuela, 1997), somnolencia diurna entre 7 y 13, ligera somnolencia diurna entre 14 y 19 puntos., la validez de consistencia interna, medido mediante el alfa de Cronbach fue de 0,83. 
También se aplicó la escala de Somnolencia excesiva diurna denominada Epworth (ESE) (Epworth Sleepiness Scale, ESS) de Johns $(1991,1992)$ (Chica $H, 2007$ ) prueba subjetiva cuyo objetivo es evaluar la magnitud de la somnolencia diurna frente a ocho situaciones de la vida diaria, indica o señala los individuos que presentaron trastornos de sueño o no y los que han sufrido privación de sueño o no, esto con un puntaje de $0=$ para Nunca; $1=$ Ligera; $2=$ Moderado, $3=$ Alta. Su puntación global presenta un rango entre 0 y 24 . De esta manera si el alumno se encuentra entre 0 y 6 puntos no presenta. Para este caso, la validez del Coeficiente de Cronbach fue de 0,85 y la correlación inter- ítem global fue de 0,51.

Con los datos obtenidos se realizó una base de datos en Microsoft Excel versión 2010, y el análisis de los datos se realizó mediante el programa estadístico SPSS versión 22. Las variables cualitativas se analizaron mediante frecuencias absolutas y porcentuales, las variables cuantitativas se analizaron con promedios, medianas y desviaciones estándar, para determinar asociación se utilizó la prueba ChiCuadrado de Pearson y Odds Ratio, con intervalos de confianza al 95\%, para controlar la confusión se utilizó un modelo de regresión logística con el método Enter. El nivel de significancia fue al 0,05.

Para controlar las variables confusoras se solicitó a los estudiantes que se abstuvieran de participar aquellos que tuvieran problemas personales o que consumieran algún fármaco. La aplicación del instrumento se realizó en forma física por parte de los investigadores en forma anónima para evitar los sesgos de respuesta. La variable dependiente objeto de estudio es el rendimiento académico y esta fue obtenida con base en el número de asignaturas reprobadas durante el curso de su carrera.

\section{Resultados}

De la aplicación de los instrumentos se obtuvieron los siguientes resultados:

Con relación a las características generales de la población, la edad promedio fue de 21,8 $\pm 3,6$ años, con un rango que oscila desde los 16 años hasta los 43 años, y la variabilidad fue medianamente homogénea $(\mathrm{CV}=16,3 \%)$. La mayor parte de la población estudiada fue de sexo femenino con $60,2 \%$, solteros $(88,3 \%)$. El estrato socioeconómico predominante fue el estrato tres con un $60,8 \%$, seguido del estrato cuatro con un $18,5 \%$ y el dos con un $15,8 \%$.

Con respecto a la condición nutricional según el IMC después de la condición normal $75 \%$, predominó el sobrepeso $15 \%$ y la obesidad $10 \%$, (siendo normal un IMC entre 18.50 - 24.99, sobre 25.00 - 29.99 y obesidad mayor de 30) no se encontró asociación significativa entre estas variables y la calidad del sueño.

Por otro lado, la aplicación de la escala de somnolencia EPWORTH y el índice de Pittsburg PSG señalan que las horas de sueño promedio fueron de 5,3 $\pm 1,2(\mathrm{Me}=5)$, con un rango de 2 a 9 horas. El promedio del índice de calidad del sueño (PSG) fue de $8,7 \pm 3(\mathrm{Me}=9)$, el mínimo fue de 1 y el máximo de 17, con respecto a la ESE el promedio fue de

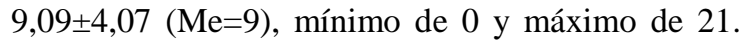
Según la escala Epworth (ESE) (Epworth Sleepiness Scale, ESS) de Johns (1991,1992) (Chica H, 2007) la calidad del sueño de los estudiantes mala (Gráfica 1), y presentan un índice de somnolencia baja.

Figura 1. Clasificación del Grado de Somnolencia de la ESE

\section{CLASIFICACIÓN DEL GRADO DE SOMNOLENCIA DE LA ESE}

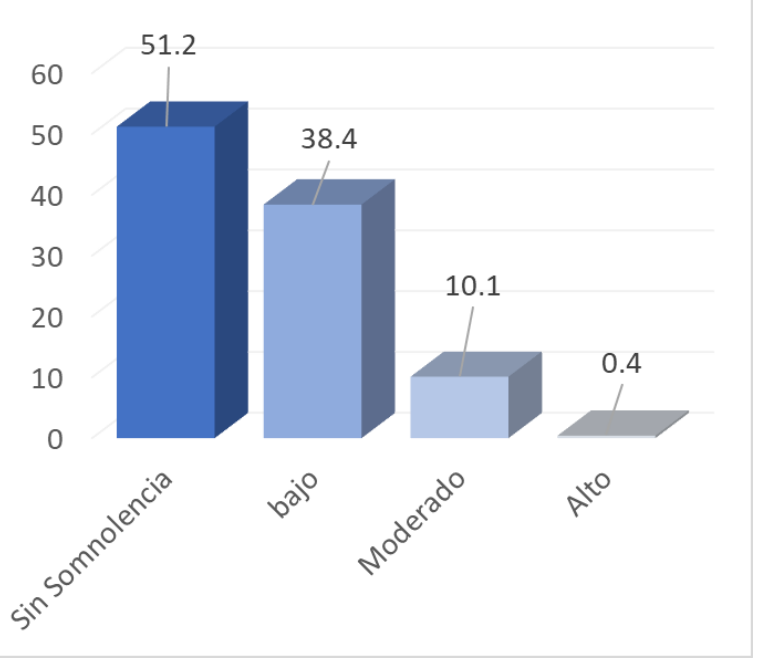


Se encontró asociación estadísticamente significativa entre el EPWORTH y PSG ( $\mathrm{p}=0,004)$. ( $\mathrm{OR}=3,01$ IC95\% (1,4 a 6,5), estudiantes con mala calidad del sueño presentaron estadios de somnolencia en $44 \%$, mientras que, para aquellos que presentaron una calidad del sueño buena o aceptable la presencia de somnolencia fue del 4\% (Tabla 1)

Tabla1. Asociación entre PSG y EPWORTH

\begin{tabular}{lccc}
\hline \multirow{2}{*}{ PSG } & \multicolumn{3}{c}{ EPWORTH } \\
\cline { 2 - 4 } & Somnolencia & Sin Somnolencia & Total \\
\cline { 2 - 4 } Malo & 111 & 103 & 214 \\
Bueno & 10 & 28 & 38 \\
Total & 121 & 131 & 252 \\
\hline
\end{tabular}

Así mismo la calidad del sueño PSG se asocia con el vínculo del estudiante a el colegio o la facultad a la que pertenece y el reprobar o no asignaturas están relacionados con la calidad del sueño y la somnolencia constituyéndose estos como un factor de riesgo para los estudiantes del colegio administrativo y jurídico ( $\mathrm{OR}=2,3$ (IC95\% 0,6 a $8,4)$ ) y para estudiantes vinculados al colegio odontológico - colegio administrativo ( $\mathrm{OR}=2,3$ (IC95\% 0,6 a 8,4) ) haber reprobado materias fue un factor protector con un OR=0,5(IC95\% 0,25 a 0,98). Esto está relacionado con la carga académica, intensidad horaria y el tiempo dedicado al desarrollo de las actividades propias de cada colegio. (Tabla 2)

Tabla 2. Distribución de PSG por Factores

\begin{tabular}{|c|c|c|c|c|c|}
\hline \multirow[b]{3}{*}{ Variables } & \multirow[b]{3}{*}{ Características } & \multicolumn{4}{|c|}{ PSG } \\
\hline & & \multicolumn{2}{|c|}{ Mala } & \multicolumn{2}{|c|}{ Buena } \\
\hline & & $\mathbf{n}$ & $\%$ & $\mathbf{n}$ & $\%$ \\
\hline \multirow{3}{*}{ Colegio* } & Jurídico & 20 & 9,0 & 4 & 10,5 \\
\hline & Administrativo & 22 & 9,9 & 10 & 26,3 \\
\hline & Odontológico & 180 & 81,1 & 24 & 63,2 \\
\hline \multirow{2}{*}{$\begin{array}{l}\text { Reprobados } \\
\text { semestres }\end{array}$} & $\mathrm{Si}$ & 27 & 12,3 & 4 & 10,8 \\
\hline & No & 192 & 87,7 & 33 & 89,2 \\
\hline \multirow{2}{*}{ Reprobado Materias* } & $\mathrm{Si}$ & 77 & 35,2 & 20 & 52,6 \\
\hline & No & 142 & 64,8 & 18 & 47,4 \\
\hline \multirow{2}{*}{$\begin{array}{l}\text { Participación de } \\
\text { actividades }\end{array}$} & $\mathrm{Si}$ & 72 & 32,6 & 12 & 31,6 \\
\hline & No & 149 & 67,4 & 26 & 68,4 \\
\hline
\end{tabular}

En estadios de somnolencia (19.4\%) reprobar semestres se constituye un factor de riesgo con EPWORTH con un OR=3,6 (1,6 a 8,5). (Tabla 3)
Tabla 3. Distribución de EPWORTH por Factores

\begin{tabular}{|c|c|c|c|c|c|}
\hline \multirow[b]{2}{*}{ Variables } & \multirow[b]{2}{*}{ Características } & \multicolumn{2}{|c|}{ Somnolencia } & \multicolumn{2}{|c|}{ Sin Somnolencia } \\
\hline & & $\mathbf{n}$ & $\%$ & $\mathbf{n}$ & $\%$ \\
\hline \multirow{3}{*}{ Colegio } & Jurídico & 15 & 11,9 & 10 & 7,6 \\
\hline & Administrativo & 12 & 9,5 & 20 & 15,2 \\
\hline & Odontológico & 99 & 78,6 & 102 & 77,3 \\
\hline \multirow{2}{*}{ Semestre } & 1 a 3 semestre & 38 & 30,2 & 37 & 28,0 \\
\hline & $\geq 4$ semestre & 88 & 69,8 & 95 & 72,0 \\
\hline \multirow{2}{*}{$\begin{array}{l}\text { Reprobados } \\
\text { semestres* }\end{array}$} & $\mathrm{Si}$ & 24 & 19,4 & 8 & 6,2 \\
\hline & No & 100 & 80,6 & 122 & 93,8 \\
\hline \multirow{2}{*}{ Reprobado Materias } & $\mathrm{Si}$ & 46 & 37,4 & 54 & 40,9 \\
\hline & No & 77 & 62,6 & 78 & 59,1 \\
\hline \multirow{2}{*}{$\begin{array}{l}\text { Participación de } \\
\text { actividades }\end{array}$} & $\mathrm{Si}$ & 39 & 31,2 & 41 & 31,1 \\
\hline & No & 86 & 68,8 & 91 & 68,9 \\
\hline
\end{tabular}

Al evaluar la calidad del sueño controlando las variables, el tener somnolencia (ESE) es un factor de riesgo para la calidad del sueño. (Tabla 4)

Tabla 4. Regresión logística de la calidad del sueño (PSG)

\begin{tabular}{lccc}
\hline & \multirow{2}{*}{ OR } & \multicolumn{2}{c}{ IC 95\% a OR } \\
\cline { 3 - 4 } Variables en el modelo & & Inferior & Superior \\
\hline Semestre perdido (Si/No) & 1.6 & 0.3 & 3.6 \\
Asignaturas perdidas (Si/No) & 0.5 & 0.2 & 1.1 \\
$\begin{array}{l}\text { Participación en actividades } \\
\text { extracurriculares (Si/No) }\end{array}$ & 1.1 & 0.5 & 2.6 \\
$\begin{array}{l}\text { Semestre (5 a 10/1 a 4) } \\
\text { Somnolencia (ESE) }\end{array}$ & 1.3 & 0.5 & 3.0 \\
$\begin{array}{l}\text { Fuente: Encuesta } \\
{ }^{p}<00,05\end{array}$ & $3.2 *$ & 1.4 & 7.4 \\
\hline
\end{tabular}

$\mathrm{Al}$ evaluar la somnolencia controlando las variables, el haber reprobado materias y el tener mala calidad del sueño (PSG) es un factor de riesgo para la somnolencia. (Tabla 5)

Tabla 5. Regresión logística de la somnolencia (ESE)

\begin{tabular}{|c|c|c|c|}
\hline \multirow{2}{*}{ Variables en el modelo } & \multirow{2}{*}{ OR } & \multicolumn{2}{|c|}{ IC 95\% a OR } \\
\hline & & Inferior & Superior \\
\hline Semestre perdido (Si/No) & $3.7^{*}$ & 1.5 & 9.5 \\
\hline Asignaturas perdidas (Si/No) & 0.7 & 0.4 & 1.3 \\
\hline $\begin{array}{l}\text { Participación en actividades } \\
\text { extracurriculares (Si/No) }\end{array}$ & 1.1 & 0.6 & 1.9 \\
\hline Semestre (5 a 10/1 a 4) & 0.8 & 0.5 & 1.5 \\
\hline Calidad del sueño (PSG) & $3.1 *$ & 1.4 & 7.3 \\
\hline
\end{tabular}


Esta investigación logró establecer relación entre la mala calidad de sueño y bajo rendimiento académico especialmente en estudiantes del colegio odontológico al ser comparados con estudiantes del colegio administrativo y jurídico de UNICOC, el porcentaje promedio de estudiantes con asignaturas reprobadas en los tres colegios es del $11.35 \%$ teniendo mayor peso porcentual la carrera de derecho con el 13\%, seguida de odontología y administración de empresas y de negocios con el $11 \%$, no obstante es importante precisar que la carga académica es mayor en la carrera de odontología y por tratarse de una disciplina del área de la salud y la rigurosidad técnico científica de la misma no la hace comparable con las ciencias jurídicas y administrativas como tal.

\section{Discusión}

Se han realizado estudios que buscan establecer la relación entre la del sueño con el rendimiento académico, no obstante al buscar la comparación con otros estudios no se encontraron investigaciones en las que se involucren estudiantes de odontología; se establece una relación con estudios basados en el área de medicina y enfocados a evaluar calidad del sueño y rendimiento académico con médicos en formación, un estudio realizado en la Universidad de Pereira con estudiantes de Medicina, halló que estos estudiantes presentan mala calidad del sueño asociándolo con bajo rendimiento académico, partiendo de características generales siendo los datos más relevantes el predomino de sexo femenino con un $60.2 \%$ (frente a un $59.4 \%$ del sexo masculino) (Cañones P.J, 2003) de la universidad Tecnológica de Pereira. Según las escalas implementadas (PSG y EPWORTH) cerca de la mitad de los estudiantes padecen baja somnolencia, a diferencia de lo publicado en el estudio comparativo en el que un $49,8 \%$ presenta somnolencia excesiva diurna. De la misma forma, los estudiantes de esta investigación fueron clasificados con mala calidad del sueño con un $85.4 \%$ (frente a un $80 \%$ ), datos similares a los reportados por la Universidad Tecnológica de Pereira en estudiantes de Medicina. (Machado-Duque \& Echeverri Chabur, 2015) Se encuentra similitud en los resultados dado que son universidades colombianas y los estudios fueron realizados con estudiantes de áreas de la salud con una alta carga académica y responsabilidad clínica. En cuanto a la carga académica y con respecto a la PSG el haber reprobado materias y el tener mala calidad del sueño es un factor de riesgo alto para presentar somnolencia teniendo en cuenta el bajo rendimiento académico, esto lo hace similar a otros estudios realizados en estudiantes de Medicina y algunas Escuelas de Educación Superior de áreas de la salud, lo importante no es solo encontrar que la relación mala calidad del sueño y rendimiento académico es causa de un desequilibrio en varios aspectos del estudiante y que involucra directamente su vida académica fomentando un posible ambiente negativo institucional sino que también es importante, el cómo crear un estado de mejora desde las instituciones, en sus planes de bienestar universitario, o seguimiento preventivo estudiantil para en futuros estudios denotar como estas políticas de mejora afectan el conjunto estudiante y que alcances la promoción y prevención como recurso universitario está influenciando en la población universitaria. Es preciso anotar que los estudiantes que formaron parte de la investigación en su mayoría es población adolescente y de acuerdo con el estudio realizado por Quevedo-Blasco, en lo referente a la calidad del sueño, se estableció que para este grupo de población, una relación directamente proporcional con el rendimiento académico, corroborando que la calidad del sueño incide de manera negativa en las calificaciones, asignaturas reprobadas y por ende en la afectación en el rendimiento académico (Blasco, 2011). Así mismo en el estudio de Guavativa Méndez y Pérez Parra (Guavativa Méndez \& Pérez Parra, 2017), se encontró que aproximadamente el $70 \%$ de los estudiantes no presentan una buena calidad de sueño y a su vez presentan algún nivel de somnolencia, se estableció además que existe una correlación entre la calidad del sueño de los estudiantes y el rendimiento académico, ya que se evidencia que entre mayor sea la puntuación del Índice de Calidad de Sueño de Pittsburgh (ICSP) menor es el rendimiento que obtienen los estudiantes, de igual modo, existe la correlación entre la Calidad de sueño y el indicie de somnolencia de Epworth, puesto que entre mayor puntuación del ICSP mayor va a ser la somnolencia que presenten los estudiantes durante el transcurso del día.

Según las escalas implementadas Epworth y Pittsburg para la Investigación, se evidencia que la mayoría de los estudiantes presentan altos niveles de somnolencia y en la clasificación de la calidad (Bohórquez, 2011) del sueño un predomino de la mala calidad de sueño, considerando un elevado nivel de riesgo para la población universitaria, 
mencionando los estados negativos anteriormente expuestos en el ser humano, desde lo emocional, social, académico, hasta conllevar esta alteración a una alteración sistémica; de igual forma el haber reprobado materias y tener mala calidad del sueño es un factor asociado a la presencia de somnolencia en estudiantes de los colegios odontológico, jurídico y administrativo - UNICOC Bogotá, lo que puede indicar que esta sea la causa de la prevalencia de bajo rendimiento académico, a su vez al evaluar la presencia de somnolencia y el haber reprobado materias y tener mala calidad de sueño es un factor de riesgo asociado a la baja somnolencia la cual fue dominante en el estudio, a futuro el nivel de somnolencia puede ser excesiva, por lo que es importante implementar políticas de promoción y prevención frente al problema, es importante prestar especial atención a los resultados generados para el colegio jurídico y administrativo que aunque la carga académica sea diferente presenta rangos estadísticamente significativos con respecto a la alteración en niveles de sueño y rendimiento académico.

El sueño y el descanso son fundamentales para tener una buena salud y lograr calidad de vida; si sólo es un factor negativo el mal dormir, al sumarle un bajo rendimiento académico que también afecta la salud, lo social, cognitivo y emocional, se puede hablar de una alerta de salud pública en este caso, que puede afectar estudiantes de odontología y de otras áreas del conocimiento. UNICOC Bogotá, no busca sólo llegar a crear ajustes en las políticas de bienestar estudiantil, sino que alerta a otras instituciones, para que logren re direccionar estrategias, frente a estas situaciones de salud pública basados en la evidencia obtenida con este estudio, donde es evidente esta importante asociación estableciendo acciones de mejora para lograr un entorno de desempeño tanto estudiantil como laboral y administrativo saludable, que conlleve a una mejora de resultados académicos, fomentando acciones de promoción de la salud y prevención de la enfermedad.

\section{Limitaciones}

Las limitaciones están relacionadas con los instrumentos utilizados, debido a que estos son subjetivos, por lo tanto, es una limitación propia de los cuestionarios, sin embargo, esto no altera la validez y la confiabilidad de los resultados obtenidos.
Con base en los resultados del estudio, sería conveniente estudiar otras variables que incidan en la calidad del sueño tales como estrés, consumo de sustancias psicoactivas y hábitos de estudio entre otras con el fin de determinar con mayor rigurosidad la relación entre la calidad del sueño y el rendimiento académico.

\section{Conclusiones}

Con lo anterior se concluye que el rendimiento académico está asociado a la mala calidad del sueño y la somnolencia las cuales fueron prevalentes, especialmente en los estudiantes del Colegio Odontológico y el Colegio Jurídico.

\section{Bibliografía}

Aguirre., R. G. (2014). Caracterización del patrón de sueño en estudiantes de la Universidad de Quintana Roo. Revista Salud Quintana Roo, 7(29), 16-20.

Ahrberg K, D. M. (2012). The interaction between sleep quality and academic performances. $\mathrm{J}$ Psychiatr Res. Journal of Psychiatric Research, 46(12), 1618-1622.

Amador J, G. V. (2015). Efectos de las horas de sueño sobre el rendimiento escolar en alumnos de nivel superior. IPN Conversus, 1-15. Obtenido de http://cuved.unam.mx/rdipycs/wp-

content/uploads/2015/08/1_Original_Amador-ycols_090914.pdf

Blasco, Q.-B. \&. (2011). Influencia del grado de somnolencia, cantidad y calidad de sueño sobre el rendimiento académico en adolescentes. ISSN 1697-2600. 2011, Vol. 11, No 1, pp. 49-65. International Journal of Clinical and Health I, 11(1), 49-65. Obtenido de https://www.google.com/search?q=20.+Queved o-Blasco+y+Quevedo-

Blasco.+Influencia+del+grado+de+somnolencia $\% 2 \mathrm{C}+$ cantidad $+\mathrm{y}+$ calidad $+\mathrm{de}+$ sue $\% \mathrm{C} 3 \% \mathrm{~B} 1 \mathrm{o}+\mathrm{s}$ obre+el+rendimiento+acad\%C3\%A9mico+en+a dolescentes.+ISSN+1697$2600 .+2011 \% 2 \mathrm{C}+\mathrm{Vol} .+11 \% 2 \mathrm{C}+\mathrm{No}+1 \% 2 \mathrm{C}+\mathrm{pp}$. $+49-65 \& \mathrm{rlz}=1 \mathrm{C} 1 \mathrm{C}$ 
Bohórquez, P. (2011). Calidad del sueño, somnolencia diurna y salud auto percibida en estudiantes universitarios. Eureka, 8(1), 80-90.

Caicedo Ochoa Y., U. G. (2016). Prevalencia del grado de somnolencia y su relación con el rendimiento académico en estudiantes de medicina de la ciudad de Tunja, Colombia, durante el año 2014. CIMEL, 20(1), 58-62.

Cañones P.J, A. J. (2003). Trastornos del sueño. Revista de la SEMG (en línea) (59), 681-690.

Cheng SH, S. C. (2012). A study on the sleep quality of incoming university students. Psychiatry Res., 197(3), 270-274.

Chica H, E. F. (2007). Validación de la Escala de Somnolencia de Epwort. Revista Salud Pública., 9(4), 558-567.

Curcio G, F. M. (2006). Sleep loss, learning capacity and academic performance. 10(5), 323-337.de la Portilla Maya S, D. L. (2019). Calidad del sueño y somnolencia diurna excesiva en estudiantes universitarios de diferentes dominios. Hacia Promoc. Salud, 24(1), 84-96.

Enríquez Guerrero, C., Segura Cardona, A., \& Cuevas, T. (2013). Risk factors related to underachievement in school-aged children in Bogotá. Investig. andina [online]., 15(26), 654666.

Escobar - Cordoba, F. e. (2011). Somnolencia diurna excesiva en estudiantes de noveno semestre de medicina de la Universidad Nacional de Colombia. Revista de la Facultad de Medicina, [S.l.], 59(3), 191-200.

Grander M.A, J. N. (2012). Sleep disturbances is associated with cardiovascular and metabolic disorders J Sleep Res. Journal of Sleep Research, 21(44), 427-433.

Guavativa Mendez, J., \& Pérez Parra, V. (2017). Relación entre calidad de sueño y rendimiento académico en estudiantes de ingeniería civil de la universidad santo tomas, sede Villavicencio. 6775.
Honorio, D., \& Hideyo, N. (2013). Estudio epidemiológico Metropolitano en Salud Mental en Lima Metropolitana y Callao-Replicación 2012. Informe General. Anales de salud Mental, 29(1), 20-22.

Lezcano H, V. Y. (2014). Características del sueño y su calidad en estudiantes de Medicina de la Universidad de Panamá. Med Cient [Internet]., 27(1), 4-10.

Lund HG, R. B. (2010). Sleep Patterns and Predictors of Disturbed Sleep in a Large Population of College Students. J Adolesc Health. 46(2), 124132.

M., G. V. (2007). Factores asociados al rendimiento académico en estudiantes universitarios, una reflexión desde la calidad de la educación superior pública. (Vol. 31). Revista Educación. Obtenido de http://www.redalyc.org/articulo.oa?id=4403110 3.

Machado-Duque, M. E., \& Echeverri Chabur, J. E.A. (2015). Somnolencia diurna excesiva, mala calidad del sueño y bajo rendimiento académico en estudiantes de Medicina. rev. colomb. psiquiatr. [online]., 44(3), 137-142.

Monterrosa Castro, A. U. (2014). Calidad del dormir, insomnio y rendimiento académico en estudiantes de medicina. Duazary, 11(2), 85-97.

Preisegolaviciute E, L. D. (2010). Associations of quality of sleep with lifestyle factors and profile of studies among Lithuanian students. Medicine (Kaunas), 46(7), 482-489. Obtenido de https://www.ncbi.nlm.nih.gov/pubmed/2096662 2

Royuela, M. J. (1997). Propiedades Clinimétricas de la Versión Castellana del Cuestionario de Pittsburgh. Vigilia Sueño. Vigilia-sueño, 9(2), 81-94.

Veldi M, A. A. (2005). Sleep quality and more common sleep- related problems in medical students. Sleep Medicine, 6(3), 269-275. 
Disomnias, desórdenes de excesiva somnolencia, desarrollo de desórdenes académicos, aprendizaje.

Villarroel V. (2014). Calidad del suelo en estudiantes de las carreras de medicina $y$ enfermería Universidad de los Andes: Mérida Venezuela (Vol. 1). Universidad Autónoma de Madrid. Departamento de Psiquiatría. Obtenido de http://hdl.handle.net/10486/663392 